Proceedings

\title{
Toward near real-time kinematics differential correction: in view of geometrically augmented sensor data for mobile microclimate monitoring ${ }^{\dagger}$
}

\author{
Stefano Tondini 1,*, Farshad Hasanabadi ${ }^{2}$, Roberto Monsorno ${ }^{1}$, and Antonio Novelli ${ }^{3}$ \\ 1 Center for Sensing Solutions, Eurac Research - Bolzano (Italy); stefano.tondini@eurac.edu \\ 2 Institute for Renewable Energy, Eurac Research - Bolzano (Italy); farshad.hasanabadi@eurac.edu \\ 3 Planetek Italia Srl - Bari (Italy); novelli@planetek.it \\ * Correspondence: stefano.tondini@eurac.edu; Tel.: +39-0471-055266 \\ + Presented at the 7th Electronic Conference on Sensors and Applications, 15 - 30 November 2020; Available \\ online: https://ecsa-7.sciforum.net/.
}

Published: 15 November 2020

\begin{abstract}
In the scenario of massive urbanization and global climate change, the acquisition of microclimatic data in urban areas plays a key role in responsive adaptation and mitigation strategies. The enrichment of kinematic sensor data with precise, high-frequency and robust positioning directly relates to the possibility of creating added-value services devoted to improving life-quality of urban communities. This work presents a low-cost cloud-connected mobile monitoring platform for multiple environmental parameters and their spatial variation in the urban context.
\end{abstract}

Keywords: position-enriched sensor data; low-cost GNSS receivers/antennas; real time kinematics; urban microclimate monitoring.

\section{Introduction}

The recent evolution of IoT devices opens up to new possibilities for urban monitoring, especially in the validation of current theoretical models and numerical simulations, as well as, to provide support and evidence in the process of decision- and policy-making [1]. In this framework, mobile microclimatic monitoring plays a crucial role, when kinematic sensors' data are enriched with precise, high-frequency and robust positioning capabilities. Indeed, spatial enrichment of moving sensors leads directly to the possibility of having fast, reliable and accurate information over time. This, in turn, allows to set up added-value services for improving the life quality of the urban communities [2,3]. In this regard, estimation methods have been tested through mobile and fixed IoT sensors for a limited spatial resolution [4].

Despite the possibility of an improved spatio-temporal resolution, standalone low-cost mobile prototypes with (i) accurate localization capacity (differential GNSS positioning capabilities) and (ii) transmitting measurements in real-time are still not common in urban and peri-urban environment.

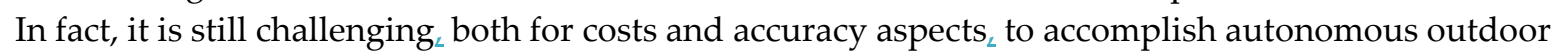
devices with reliable positioning, as recent studies have pointed out $[5,6]$. In this sense, low-cost Global Navigation Satellite System (GNSS) receivers can implement improved georeferencing capabilities [7] by exploiting GNSS receivers featuring multifrequency and multi-constellation capabilities. Recently, the advancements in low-cost chipset development have resulted in the commercialization of low-cost high-precision GNSS receivers off the shelves. Furthermore, there are advantages of using such GNSS receivers like lower power intensity, portable size, and high integration, to name a few [8]. 


\section{Materials and methods}

In this work, a preparatory study to assess the positioning capabilities of a low-cost GNSS receiver is proposed in view of microclimatic sensors' data spatial enrichment.

The hardware is based on a U-blox ${ }^{\circledR}$ C099-F9P application board [9] set as the low-cost GNSS receiver, coupled with a Raspberry Pi (RPi) [10]. The prototype has been benchmarked against a standard high-accuracy (geodetic) GNSS configuration from Leica ${ }^{\circledR}$ composed by a AS10 antenna, GS10 receiver and CS15 controller. The open-source suite for precise positioning RTKlib has been exploited both to log the U-blox data on the prototype, and to postprocess the different positioning solutions tested [11]. RTKlib suite has been chosen because its multiplatform capabilities, the high number of parameters that can be tuned to get to the optimal configuration ${ }_{\llcorner}$and because it is also easy to encompass it in an automatic data acquisition chain.

\subsection{U-blox based prototype}

A picture of the low-cost prototype inner components is shown in Figure 1. Even though the Ublox ${ }^{\circledR}$ C099-F9P board by itself has good capabilities, we decided to give more flexibility and computational power to the prototype by coupling it with a RPi. The connection of the GNSS module to the RPi has been achieved though serial communication. The RPi as a development platform allows for many possible upgrades of the prototype. Among the others, the chance to exploit a $3 / 4 \mathrm{G}$ expansion shield for cellular connectivity or the availability of many connection buses to couple the prototype with other sensors (temperature/relative humidity and solar radiation in the picture). The system is powered by a 5V- $15000 \mathrm{mAh}$ USB power bank, which ensures long lasting operation capabilities and portability, once fully charged. All the hardware components, besides the ANN-MB00 multi-band GNSS antenna and ground plate, have been enclosed within an IP68 case.

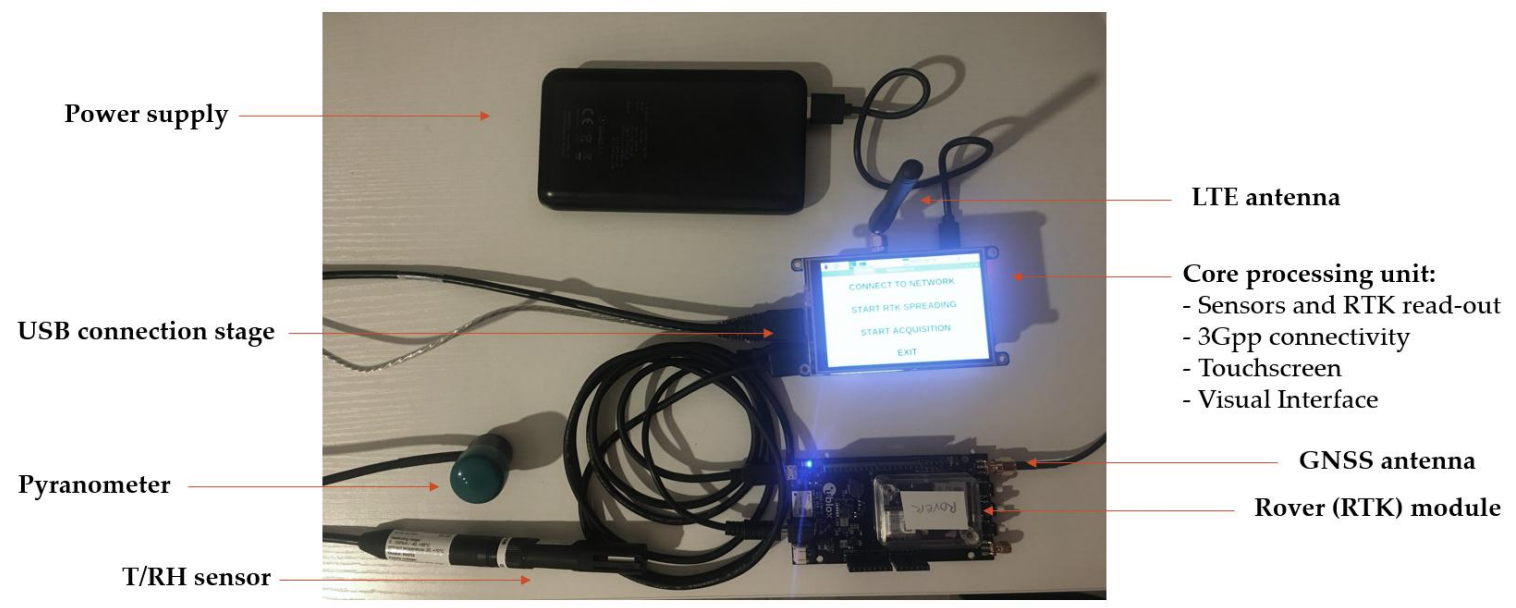

Figure 1. Schematics of the building blocks of the low-cost prototype that has been involved into the tests. The core processing unit is made of a RPi 4 couple with a SIM800 GSM expansion shield, and an Adafruit PiTFT 3.5" touch screen display. The GNSS roved module is a U-blox C099-F9P application board. The sensors shown in the picture have not been used for the purposes of this paper.

\subsection{Filed tests and data processing workflow}

Figure 2 shows the filed campaign setup (left) and the three different configurations tested (right). A LEICA ${ }^{\circledR}$ tripod has been used as steady support for devices put in base mode, while city bicycle has been used as rover carrier for the U-blox ${ }^{\circledR}$ based prototypes. Each configuration has been tested for approximately 1 hour of continuous acquisition where the devices have been set to get GNSS data at $5 \mathrm{~Hz}$. To get differential corrections for configuration \#1 and \#3, RINEX data from a permanent base station of the STPOS network managed by the Geodetical Office of the Autonomous province of Bolzano have been exploited [12]. 

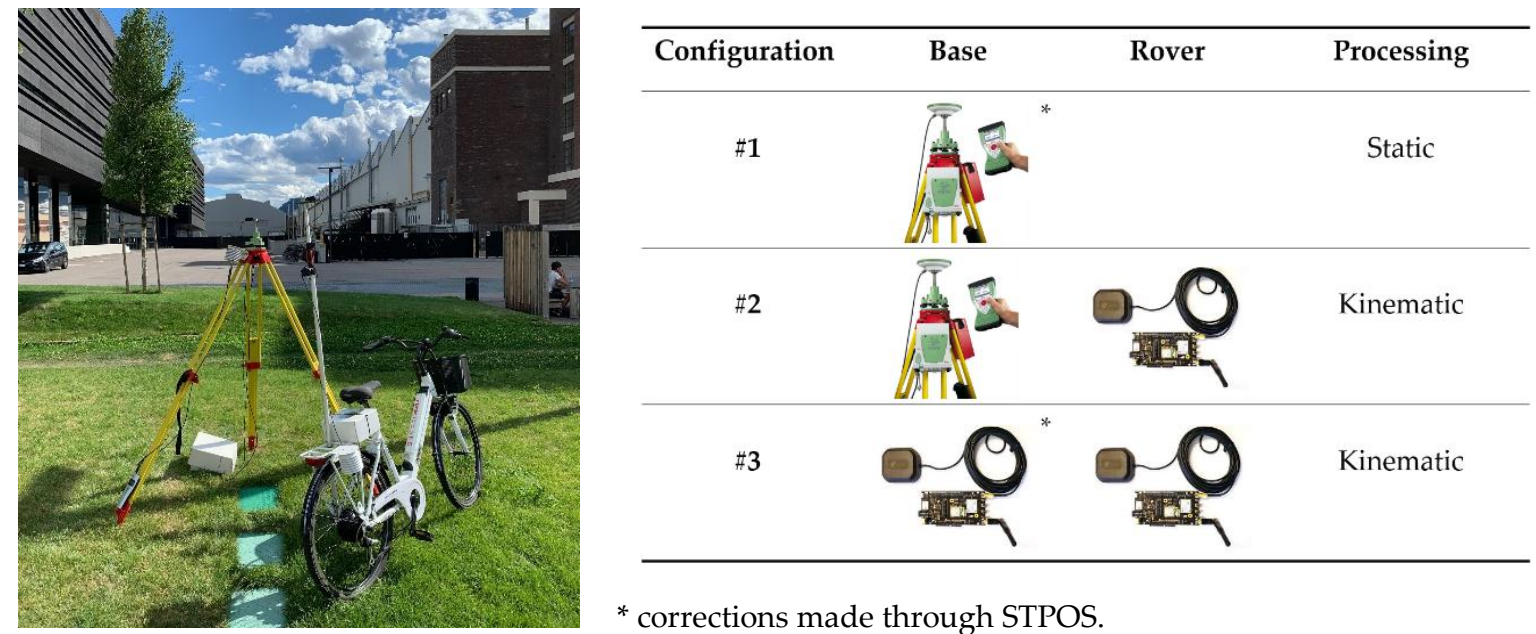

Figure 2. (left) Picture of the LEICA support and the bicycle that have been used during the field tests to carry the base and the rover devices, respectively. (right) Table of the different configuration under test. Due to limited equipment availability, the configurations have been executed in succession.

To run the experimental acquisition, the LEICA ${ }^{\circledR}$ system has been operated through the proprietary software SmartWorx Viva and Geo Office has been used to convert the data to RINEX format. Whereas the command line interface (CLI) program STR2STR (part of RTKlib) has been compiled in the NOOBS environment (on RPi) to log the raw GNSS data from the U-blox ${ }^{\circledR}$ based prototype, and RTKCONV has been used to convert receivers' raw data to RINEX format [13]. Lastly, the RTKPOST has been exploited in each configuration to provide positioning solutions from the collected data after conversion to RINEX. RTKlib supports many positioning solutions with GNSS for both real-time-kinematic (RTK) and post-processed solution with differential correction. To accomplish the post processing, the following steps have been performed:

1. download of the RINEX file from the nearest STPOS base station to the test site and covering the whole duration of the measurement session;

2. post-processing calculation of the coordinates of the LEICA ${ }^{\circledR}$ station (static point differential positional solution);

3. coordinates obtained at point 2. are used to retrieve the positions for the first kinematic session, i.e. configuration $\# 2$, with the U-blox $₫$ based rover. In particular, LEICA ${ }^{\circledR}$ measurements are used both to retrieve the base coordinates (i.e. related to tripod position) and then the base coordinates are used to retrieve the rover coordinates in the differential solution;

4. coordinates obtained at point 2 are used when performing calculations with the RINEX files acquired from the two U-blox ® based prototypes (configuration \#3).

\section{Results}

The field tests have taken place in the industrial district of the city of Bolzano (IT). The LEICA ${ }^{\circledR}$ high-accuracy GNSS set has been placed in the inner courtyard of the NOI Technology Park, far from typical GNSS source of disturbances. This setup has been kept fixed during the tests of the \#1 and \#2 configurations. For configuration \#3, the AS10 antenna on the LEICA $₫$ tripod has been replaced with the ANN-MB-00 multi-band GNSS antenna connected to one of the two U-blox ${ }^{\circledR}$ receivers in base configuration mode. The other U-blox ${ }^{\circledR}$ based prototype has been mounted on an electric bike provided of proper support for the antenna (the aluminum pole in Figure 2) in order to place the antenna in a position not affected by the head of the driver during the tests. According to this experimental design, kinematic tests (involving moving rover) were performed only in the \#2 and \#3 tests. In the following section, the results obtained from the three configurations sketched in Figure 2 are reported and discussed. 


\subsection{Configuration \#1}

Figure 3 shows the Ground Track plot (left) and the Skyplot view of the satellites (right) obtained with RTKplot after the post-processing of the first dataset from the LEICA ${ }^{\circledR}$ GNSS system.

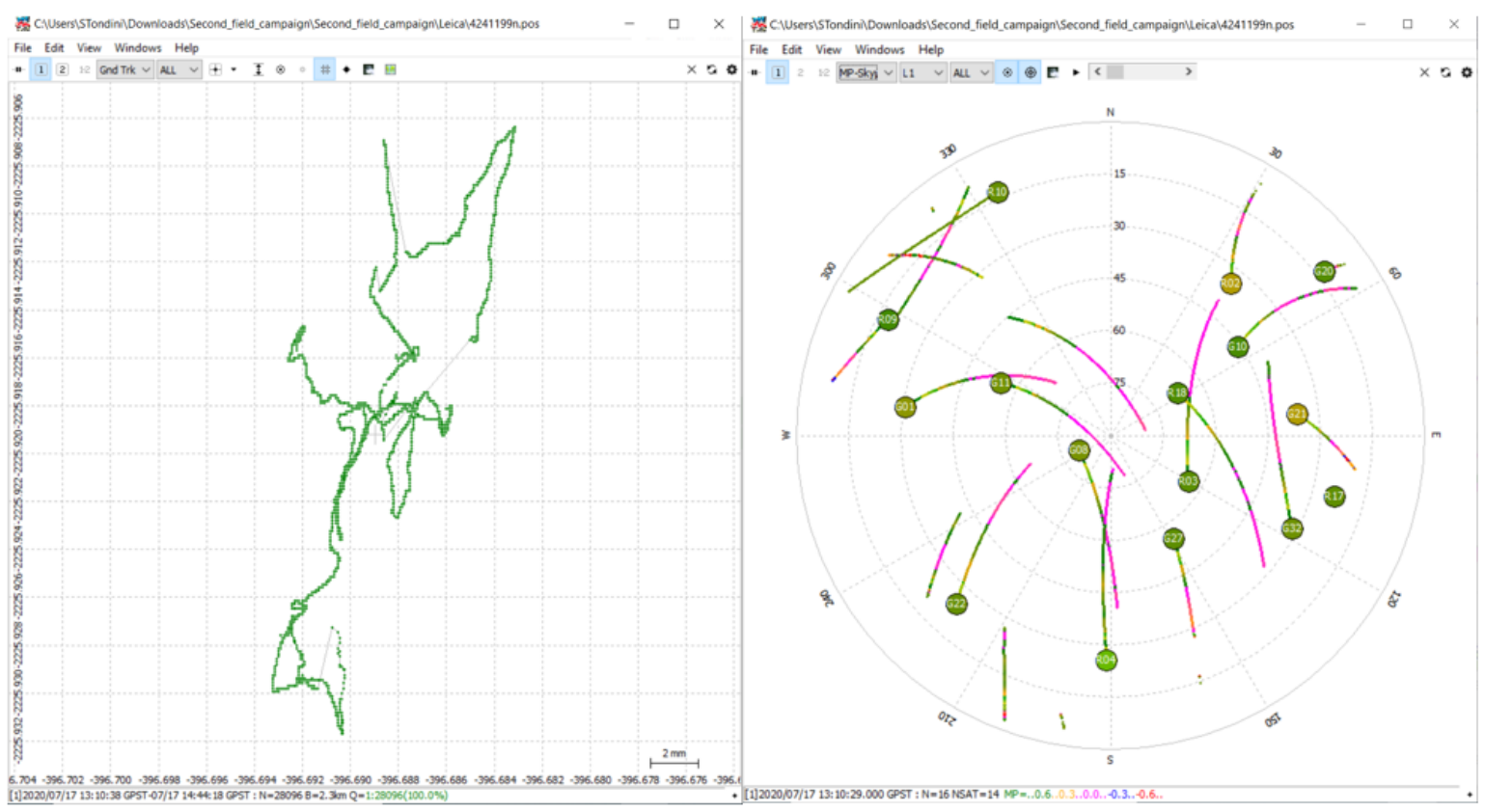

Figure 3. (left) Ground Track plot and (right) MP-Skyplot of the configuration \#1.

\subsection{Configuration $\# 2$ and $\# 3$}

The field survey paths performed with the rover in $\# 2$ and $\# 3$ configurations are shown in Figure 4. The green and yellow dots represent the fixed and floating positional solutions, respectively.

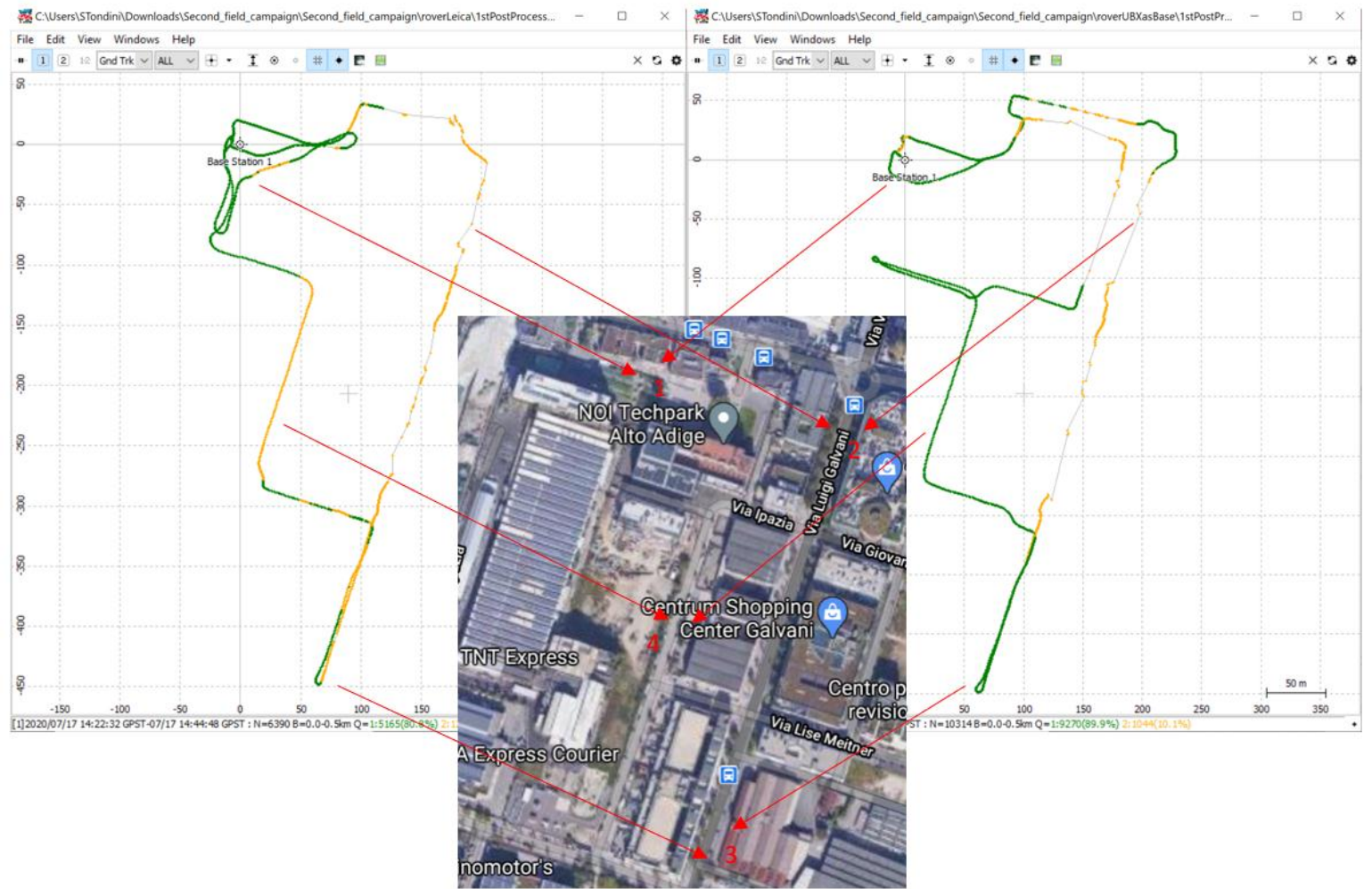

Figure 4. Ground Track plots of the rover surveys in the \#2 configuration (left) and \#3 configuration (right). The Google Maps view of the surroundings of the NOI Technology park is shown as inset. 


\subsection{Outcomes discussion}

Configuration \#1 is showing 100\% fixed solutions, as it is expected from such a setup. During the test, GPS (US), GALILEO (EU), GLONASS (Russia) and BEIDOU (China) constellations were acquired. This allowed a good availability of satellites and redundancy. The post processing (see Section 2.2 - point list 2) has led to the coordinates: lat. 46.4790, long 11.3316 and height $298.9675 \mathrm{~m}$. Standard deviations as from the .pos file obtained through RTKPOST are reported in Table 1.

Table 1. Standard deviation of positional solution from \#1 configuration in ENU (east/north/up) coordinates.

\begin{tabular}{ccc}
\hline sdE (m) & sdN (m) & sdU (m) \\
\hline 0.00011 & 0.00016 & 0.00036 \\
\hline
\end{tabular}

The coordinates achieved can be converted using the cartographic reference system UTM32N (EPSG:6707) through the PyProj python library [14].

The ratio between fixed/floating solutions has been considered as an indicator of the performance for the configuration with differential corrections ( \#2 and \#3). From a rough comparison between the two tests we see that the $\# 2$ configuration has achieved $80.8 \%$ of fixed solutions, while configuration \#3 has achieved $89.9 \%$ fixed solutions. Despite this notorious imbalance, we cannot assess that one configuration is better than the other for at least because the tests have not been carried out at the same time and following the exact same path. However, some common behaviors, encountered in both tests referring to the numbering in the inset of Figure 4, can be highlighted:

- (point 1) a fast convergence toward fixed solutions is reached in the nearby the base station, due to the open sky condition within the NOI Techpark courtyard and the low driving speed allowed;

- (point 2) the longest straight path on the right side of the Ground Track plots is the one that with the biggest amount of un-fixed solutions, due to the fact that the cycle lane is mostly covered by the tree canopy and is close to building's walls (which is preventing a good signal to be acquired);

- (point 3) even with limited sky view factor, the amount of fixed solutions increases rapidly with decreasing the speed of the rover, at the about-turn point for instance;

- (point 4) multipath effects can be also detrimental turning into floating solutions when driving along buildings and canyon walls.

However, the preliminary tests carried out show that similar accuracy can be reached in nearreal time kinematics even when a low-cost base station is involved to get differential correction.

\section{Conclusions}

The achieved results confirm the approach is suitable for urban monitoring mobile applications, where several nodes can be installed, for instance, on public transport vehicles (commercial average speed below $40 \mathrm{~km} / \mathrm{h}$ ) to expand the spatio-temporal resolution (centimeter-level accuracy in seconds) of environmental data, while keeping low the number of sensors to deploy. Major accuracies might be expected using low-cost devices with inertial measurement unit (IMU) capabilities.

The outlook is the assessment of statistic correlations between local microclimatic conditions, urban surface usage and urban morphology to aid the development of sustainable energy and climate action plans at the municipality level.

Author Contributions: Conceptualization, A.N.; methodology, A.N. and S.T.; software, F.H.; validation, S.T. and F.H.; formal analysis, F.H.; investigation, S.T.; resources, S.T.; data curation, F.H.; writing-original draft preparation, S.T.; writing-review and editing, F.H., A.N. and R.M.; visualization, S.T.; supervision, R.M.; project administration, S.T.; funding acquisition, R.M. All authors have read and agreed to the published version of the manuscript. 
Funding: The research leading to these results has received funding from the European Regional Development Fund, Operational Programme Investment for growth and jobs ERDF 2014-2020 under Project number ERDF1094, Data Platform and Sensing Technology for Environmental Sensing LAB - DPS4ESLAB.

Acknowledgments: The authors would like to thank the NOI Techpark facility management to have eased the experimental campaigns inside and outside the Technology Park area.

Conflicts of Interest: The authors declare no conflict of interest. The funders had no role in the design of the study; in the collection, analyses, or interpretation of data; in the writing of the manuscript, or in the decision to publish the results.

\section{References}

1. Argüeso, D.; Evans, J.P.; Pitman, A.J.; Di Luca, A. Effects of City Expansion on Heat Stress under Climate Change Conditions. PLoS ONE 2015, 10, e0117066, doi:10.1371/journal.pone.0117066.

2. Khashoggi, B.F.; Murad, A. Issues of Healthcare Planning and GIS: A Review. ISPRS Int. J. Geo-Inf. 2020, 9, 352, doi:10.3390/ijgi9060352.

3. Liu, Q.; Ullah, H.; Wan, W.; Peng, Z.; Hou, L.; Qu, T.; Ali Haidery, S. Analysis of Green Spaces by Utilizing Big Data to Support Smart Cities and Environment: A Case Study About the City Center of Shanghai. ISPRS Int. J. Geo-Inf. 2020, 9, 360, doi:10.3390/ijgi9060360.

4. Hayashi, S.; Takahashi, H.; Abe, T.; Chiba, S.; Suganuma, T. An Estimation Method of Urban Microclimate Using Mobile IoT Environment Sensors. In Proceedings of the 2019 IEEE 8th Global Conference on Consumer Electronics (GCCE), Osaka, Japan, 15-18 October 2019; pp. 642-643.

5. Pulighe, G.; Baiocchi, V.; Lupia, F. Horizontal accuracy assessment of very high-resolution Google Earth images in the city of Rome, Italy. Int. J. Digital Earth 2016, 9, 342-362, doi:10.1080/17538947.2015.1031716.

6. Aguilar, M.A.; Nemmaoui, A.; Aguilar, F.J.; Novelli, A.; García Lorca, A. Improving georeferencing accuracy of Very High Resolution satellite imagery using freely available ancillary data at global coverage. Int. J. Digital Earth 2017, 10, 1055-1069, doi:10.1080/17538947.2017.1280549.

7. Robustelli, U.; Baiocchi, V.; Pugliano, G. Assessment of Dual Frequency GNSS Observations from a Xiaomi Mi 8 Android Smartphone and Positioning Performance Analysis. Electronics 2019, 8, 91, doi:10.3390/electronics8010091.

8. Liu, T.; Li, B. Single-frequency BDS/GPS RTK with low-cost U-blox receivers. In Proceedings of the 2017 Forum on Cooperative Positioning and Service (CPGPS), Harbin, China, 19-21 May 2017; pp. 232-238.

9. C099-F9P Application Board. Available online: https://www.u-blox.com/en/product/c099-f9p-applicationboard (accessed on 14 October 2020).

10. Raspberry $\mathrm{Pi}$ 4. Available online: https://www.raspberrypi.org/products/raspberry-pi-4-model-b/ (accessed on 14 October 2020).

11. RTKLIB: An Open Source Program Package for GNSS Positioning. Available online: http://www.rtklib.com/ (accessed on 14 October 2020).

12. STPOS South Tyrolean Position Service. Available online: http://www.provincia.bz.it/it/servizi-az.asp?bnsv_svid=1008891\#accept-cookies (accessed on 14 October 2020).

13. RTKLIB ver. 2.4.2 Manual. Available online: http://www.rtklib.com/prog/manual_2.4.2.pdf (accessed on 14 October 2020).

14. pyproj Documentation-pyproj 2.6.1 Documentation Available online: http://pyproj4.github.io/pyproj/stable/ (accessed on 14 October 2020).

Publisher's Note: MDPI stays neutral with regard to jurisdictional claims in published maps and institutional affiliations.

(C) 2020 by the authors. Submitted for possible open access publication under the terms and conditions of the Creative Commons Attribution (CC BY) license (http://creativecommons.org/licenses/by/4.0/). 